\title{
On the Complexity of Bilinear Forms*
}

\author{
Noam Nisan ${ }^{\dagger}$ \\ Institute of Computer Science, \\ Hebrew University of Jerusalem, Israel.
}

\author{
Avi Wigderson $\ddagger$ \\ Institute of Computer Science, \\ Hebrew University of Jerusalem, Israel.
}

\begin{abstract}
This paper provides some new lower and upper bounds on computing bilinear forms by arithmetic circuits. The complexity measures considered are circuit size, formula size and time-space trade-offs.
\end{abstract}

\section{Introduction}

This paper is concerned with the arithmetic complexity of bilinear forms. Such a function $f(x, y)$ over vectors of variables $x, y$ is naturally defined by a matrix $M$ with $f(x, y)=y M x$. Note that the same $M$ also defines a set of linear forms in the $x$ variables, namely $M x$. This paper contains several new bounds on the complexity of computing such functions. The complexity measures of interest are circuit and formula size, as well as time-space trade-offs.

In the second section of the paper we are interested in general computation over arbitrary

\footnotetext{
${ }^{*}$ This work was done while the authors visited BRICS at the University of Aarhus, Denmark

${ }^{\dagger}$ This work was supported by USA-Israel BSF grant 92-00043 and by a Wolfeson research award administered by the Israeli Academy of Sciences.

${ }^{\ddagger}$ This work was supported by USA-Israel BSF grant 92-00106 and by a Wolfeson research award administered by the Israeli Academy of Sciences.
}

fields, and focus on the smallest product $T S$ of time and space required. Our main result here is proving that for every field and every $n$ there are $n \times n$ matrices $M$ for which computing the linear forms $M x$ requires nearly quadratic $T S$, while the bilinear form $y M x$ can be computed in nearly linear $T S$. The proof uses small, depth 2 superconcentrators to construct matrices $M$ in which all minors have high rank.

The motivation for this result comes for a question of Borodin [Bo94], who asked if there is an space-efficient implementation of the BaurStrassen theorem [BS82]. This remarkable theorem asserts that the time (or size) it takes to compute all $n$ partial derivatives (one for each variable) of a polynomial $f$ is only larger by a constant factor then the time to compute $f$ itself. Thus time lower bounds on computing $n$ functions can (and do) yield lower bounds for computing a single function.

One frustration of (both arithmetic and Boolean) circuit complexity is that we have quadratic lower bounds on $T S$ for computing $n$ functions (even $n$ linear functions) on $n$ variables, but no similar bounds are known for computing a single one. Observe that the set of linear functions $M x$ is the set of derivatives according to the $y$ variables of the single bilinear form $y M x$. Thus an analog of the Baur-Srassen construction, which is efficient in the $T S$ measure, would resolve that particular frustration. However, the result stated above shows that no such analog can exist. In fact, in contrast to time complexity, the gap in time space product between computing a function and computing all its derivatives is almost $n$, as bad as possible. 
Despite the above, we can give some weak lower bounds on time-space trade-offs, for the same bilinear forms used to exhibit the gap above. We show that they require $2^{T / n} T S=\Omega\left(n^{2}\right)$. This follows a simple adaptation of the AlonMaass technique ([AM88]), originally devised for oblivious branching programs.

In section 3 we switch gears and move to discuss circuits over the real or complex fields, which can use only bounded constants in the computation. This may be a severe restriction. However, considering this model has several motivations. First and foremost, we have no superlinear lower bounds even on formula size of bilinear forms in the general model, so we might as well start with this restricted one. Second, it was suggested as a natural model (for computing linear functions) by Morgenstern [Mo73] and Chazelle [Ch94]. Morgenstern observes that natural algorithms like FFT use only small constants, and actually proves that under this restriction the $n \log n$ time bound it is optimal. Chazelle argues that for algorithms in comutational geometry, the finite representation of numbers is essenntially equivalent to bounded coefficients, and then proves similar $n \log n$ lower bounds in this model for geometric range-query problems.

The above mentioned results apply only to computing many (linear) functions. We provide lower bounds on the circuit and formula size of single explicit bilinear form, e.g. the one associated with any Hadamard or Discrete Fourier Transform matrices.

We first obtain an $\Omega(n \log n)$ circuit lower bound. It follows a simple observation that the afore mentioned Baur-Strassen construction can be carried over in the restricted model, and thus the above results of, e.g., Morgenstern [Mo73] imply it directly.

We then obtain an $n^{5 / 4}$ lower bound on the formula size for these bilinear forms, as well as $n^{1+\epsilon}$ lower bounds for other explicit matrices, arising from projective geometries and other block designs. The proof uses the Hoffmann-Wielandt Inequality, which bounds the effect of perturbations on the eigenvalues of a matrix.

\section{Definitions and Basic Facts}

\subsection{Circuits}

Let $\mathrm{F}$ be a field, and $X=\left\{x_{1}, x_{2}, \cdots, x_{n}\right\}$ be indeterminates. A circuit over $F$ with variables $X$ is a directed acyclic graph whose input nodes are labeled with the elements of $X$. Every internal node of the circuit computes either a + gate or a $\times$ gate. $\mathrm{A}+$ gate, with arbitrary coefficients from $F$ on its input wires, computes that linear combination of its inputs. A $\times$ gate computes the product of its inputs. (Note that we explicitely allow unbounded fan-in.) Thus every node computes a polynomial in $F[X]$. A polynomial $f \in F[X]$ is said to be computed by a circuit $A$ if it is computed by one of the nodes of $A$.

The most basic complexity measure of a function is its circuit size. The size of a circuit is the number of edges it has. For any polynomial $f$ we denote by $C(f)$ the size of the smallest circuit computing $f$. This notion is extended to computing several polynomials: $C\left(f_{1}, f_{2}, \cdots, f_{m}\right)$ is the size of the smallest circuit computing them all.

Note: Technically, for the measure $C$ and other measures we will define, we will distinguish the field $F$ over which the circuit is computing by the notation $C_{F}$. However, as $F$ will almost always be clear from the context, we shall ususally omit this subscript.

Another fundamental measure is the depth of a circuit $A$, denoted $D(A)$, which is the length of the longest directed path in $A$. The depth complexity of a polynomial $f, D(f)$, is the smallest depth of a circuit computing $f$ (and similarly for computing many polynomials).

Two restricted notions of circuits give rise to two other complexity measures: formula size and time-space tradeoffs.

A formula is a circuit in which every node has at most one outgoing edge (fan-out 1), thus the graph is a tree. The formula size of $f, L(f)$ is the size of the smallest formula computing $f$.

Any circuit can be "implemented" by a straightline program over the input variables and a set of "registers" variables. Such a program is a sequence of instructions $X \leftarrow Y \circ Z$, with $\circ \in$ 
$\{+, \times\}^{1}, X$ a register, and each of $Y, Z$ being either a register of input variable. Such a program $A$ computes all polynomials that occupy a register at some point in the program. Its space $S(A)$ is the total number of different register used, and its time is the total number of instructions.

For any polynomial $f$ denote by $T S(f)$ the smallest value of the product of $T(A) \times S(A)$ over all programs $A$ computing $f$. For several functions $T S\left(f_{1}, \cdots, f_{m}\right)$ is similarly defined.

Two simple relations between these measure are:

Proposition 1 - For every $f, C(f) \leq T S(f) \leq$ $C(f)^{2}$.

- If $f$ is computed by a formula $A$, then $T S(f) \leq$ $C(A) D(A)$.

\subsection{Derivatives}

Let $f \in F[X]$ be a polynomial. Denote by $\Delta f$ the set of $n$ partial derivatives of $f$ according to each of the variables in $X$. This is a set of polynomials in $F[X]$ and it is natural to relate its circuit complexity to the of $f$. It is not hard to see that each derivative can be computed with size $C(f)$, so for every $f, C(\Delta f) \leq n C(f)$. A remarkable theorem of Baur and Strassen [BS82] (see a simple proof by Morgenstern [Mo85]) says that there is no need to lose the factor of $n$.

Theorem 1 [BS82] For every $f, C(\Delta f) \leq 3 C(f)$.

This theorem was used in [BS82] to convert lower bounds for computing a set of polynomials into a lower bound for a single function. For example, Strassen's lower bound [St73] $C\left(x_{1}^{n}, x_{2}^{n}, \cdots, x_{n}^{n}\right)=$ $\Omega(n \log n)$ immediately implies that $C\left(x_{1}^{n}+x_{2}^{n}+\right.$ $\left.\cdots+x_{n}^{n}\right)=\Omega(n \log n)$.

\subsection{Linear functions}

Let $\mathrm{M}$ be an $n \times n$ matrix with entries in $F$. We associate with $M$ the vector of $n$ linear functions $l_{M}$ on variables $X$ defined by the matrix vector product $l_{M}(\bar{x})=M \bar{x}$, where $\bar{x}=\left(x_{1}, x_{2}, \cdots, x_{n}\right)$.

\footnotetext{
${ }^{1}$ Again, + has arbitrary coefficients
}

Clearly, every $M$ can be computed by linear circuits, i.e. circuits that do not use multiplication. Let $C^{l}\left(l_{M}\right)$ denote the size of the smallest linear circuit computing $l_{M}$. While it is open whether using multiplications can be significantly useful over finite fiels, it is easy to see that over infinite fields it cannot.

Proposition 2 For every infinite field $F$, and every matrix $M, C^{l}\left(l_{M}\right)=C\left(l_{M}\right)$.

While simple counting shows that for most matrices $M, C\left(l_{M}\right)$ is nearly quadratic in $n$, it is a major open problem to exhibit an explicit infinite family of matrices for which $C\left(l_{M}\right)$ is superlinear. However, for the $T S$ measure such bounds can be obtained, via the elegant sufficient condition of Valiant [Va76], (see also Tompa [To80]).

\section{Theorem 2 [ $V a 76]$}

- Let $M$ be an $n \times n$ matrix in which every square submatrix is nonsingular. Then $T S\left(l_{M}\right)=\Omega\left(n^{2}\right)$.

- Let $M$ be an $m \times n$ matrix with $m \leq n$. Assume that for every $s \leq m$, every $s \times$ $(n-m / 2)$ submatrix of $M$ has rank at least $s / 2$. Then $T S\left(l_{M}\right)=\Omega\left(m^{2}\right)$.

We conclude by remarking that there are many explicit families of matrices with all minors nonsingular, and thus quadratic lower bounds on $T S\left(l_{M}\right)$ are available. Examples include all $n \times n$ matrices $\mathrm{M}$ of the form:

- $M_{i j}=\omega^{i j}$, with $\omega$ a $p$ th root of unity, and $p>n$ a prime.

- $M_{i j}=1 /(i+j)$.

\subsection{Bilinear functions}

Again let $M$ be a matrix over $F$. We associate with it the bilinear form $b_{M}$ over the $2 n$, variables $\left\{x_{1}, \cdots, x_{n}, y_{1}, \cdots, y_{n}\right\}$ by defining $b_{M}(\bar{x}, \bar{y})=$ $\bar{y} M \bar{x}$.

A bilinear circuit on variables $\left\{x_{1}, \cdots, x_{n}, y_{1}, \cdots, y_{n}\right\}$ is one in which every multiplication gate has exactly two inputs, one of which is a linear function 
of the $x$ variables and the other a linear function of the $y$ 's. Again it is clear that every bilinear function is computed by such a circuit, and we denote by $C^{b}\left(b_{M}\right)$ the size of the smallest bilinear circuit for $b_{M}$.

Again, it is not known if significant savings are possible in general using arbitrary circuits for computing bilinear forms, but for infinite fields they don't.

Proposition 3 For every infinite field $F$ and every matrix $M, C^{b}\left(b_{M}\right)=O\left(C\left(b_{M}\right)\right)$.

Now observe that for any matrix $M$, the set of linear functions $l_{M}$ is a subset of the derivatives $\Delta b_{M}$ of the bilinear function $b_{M}$. Thus, an immediate corollary to Theorem 1 is

Corollary 1 For every matrix $M$ over any field, $C\left(l_{M}\right) \leq 3 C\left(b_{M}\right)$.

In a similar way to bilinear fcircuits we define bilinear formulae, and $L^{b}\left(b_{M}\right)$. It is interesting to note that no analog of proposition 3 is known for this measure. Note that bilinear formulae have a very simple structure - without loss of generality they have depth 3: a sum of $(t$, say) products of two linear forms. Formally, any such formula for $b_{M}$ gives the decomposition:

Equation $1 M=\sum_{i=1}^{t} M_{i}$, with $M_{i}=\bar{u}^{i} \otimes \bar{v}^{i}$.

Here $\otimes$ denotes exterior product and thus each $M_{i}$ has rank 1. The size of the formula with this decomposition is simply the total number of nonzero entries in all vectors $\bar{u}^{i}, \bar{v}^{i}$. Equivalently, setting $U$ to be the $n \times t$ matrix whose columns are $\bar{u}^{i}$, and $V$ the $t \times n$ matrix whose rows are $\bar{v}^{i}$, we have

\section{Equation $2 M=U V$}

Again, the size of the formula giving this decomposition is the total number of nonzero entries in $U$ and $V$.

\subsection{Superconcentrators}

For a dircted acyclic graph $G(V, E)$ denote by $I$ (resp. $O$ ) the subset of vertices $V$ with indegree (resp. outdegree) 0, and call them inputs (resp. outputs). The size of $G, C(G)$, is its number of edges, and the depth of $G, D(G)$ is the length of the longest directed path in $G$ (necessarily between from an input to an output).

$G$ is called an $n$-superconcentrator if $|I|=$ $|O|=n$, and for every $k$ and every two subsets $I^{\prime} \subseteq I$ and $O^{\prime} \subseteq O$ with $\left|I^{\prime}\right|=\left|O^{\prime}\right|=k$ there are $k$ vertex disjoint paths from $I^{\prime}$ to $O^{\prime}$ in $G$.

Let $c_{d}(n)$ denote the size of the of the smallest $n$-superconcentrator of depth $d$. Determining the functions $c_{d}$ for various values of $d$, as well as finding explicit small and shallow superconcentrators has been a major object of study [Pi78, Pi82, DDPW]. We shall need the following two upper bounds on the size of superconcentrators of depth 2, the first being nonconstructive and the second explicit.

Theorem 3 [Pi82] $c_{2}(n)=O\left(n(\log n)^{2}\right)$.

Theorem 4 [WZ93] There is a polynomial time algorithm which for every $n$ outputs an n-superconcentrator of depth 2 and size $n^{1+o(1)}$. (The best bound on the o(1) term is actually $(\log n)^{-1 / 2}$ [SZ94].)

In any depth 2 graph $G$, the vertices decompose to three sets, $V=I \cup R \cup O$, which are resp. the inputs, middle vertices, and outputs (for any direct edge between $I$ and $O$ we can insert a new vertex to split it in two, without effecting the size by more than a factor of 2). Let $|I|=|O|=n$ and $|R|=t$. Any assignment of weights $w$ (from a field $F$ ) to the edges of $G$ (i.e. $w: E \rightarrow F)$, naturally defines a matrix over $F$ as follows. First, replace every non-edge of $G$ by an edge of weight 0 . Then, for every $i \leq t$ define two $n$-vectors $\bar{u}^{i}$ (resp. $\bar{v}^{i}$ ) by $\bar{u}_{k}^{i}\left(\right.$ resp. $\left.\bar{v}_{k}^{i}\right)$ is the weight on the edge $\left(I_{k}, R_{i}\right)$ (resp. $\left(R_{i}, O_{j}\right)$. Define $M_{i}=\bar{u}^{i} \otimes \bar{v}^{i}$, and $M=M(G, w)=\sum_{i=1}^{t} M_{i}$. This gives a decomposition as in Equation 1, and thus the fomula size upper bound:

Lemma 1 For every depth 2 graph $G$ and weights $w, L^{b}\left(b_{M(G, w)}\right) \leq|E|$.

\subsection{Schwartz's Lemma}

An extremely useful lemma regarding the zeros of multivariate polynomials was proved by Schwartz [Sc80]. 
Theorem 5 [Sc80] Let $f \in F[X]$ be a polynomial of total degree $d$, which is not identically zero. Let $H$ be any finite subset of $F$. Let $w$ : $X \rightarrow H$ be a random function, assigning to each variable an independent random, uniformly distributed element of $F$. Then $\operatorname{Pr}[f(w)=0] \leq$ $d /|H|$.

\subsection{Algebra}

For this section our field is the reals $R$. For $\bar{v} \in$ $R^{n}$, let

- $|\bar{v}|=\sum_{i=1}^{n}\left|v_{i}\right|$ be the $l_{1}$ norm of $\bar{v}$,

- $\|\bar{v}\|=\left(\sum_{i=1}^{n} v_{i}^{2}\right)^{\frac{1}{2}}$ be the $l_{2}$ norm of $\bar{v}$

- $|\bar{v}|_{\infty}=\max _{i}\left|v_{i}\right|$ be the $l_{\infty}$ norm of $\bar{v}$.

Let $A$ be an $n \times m$ matrix. $|A|,|A|_{\infty}$ and $\|A\|$ will denote the norms of $A$ viewed as an $n \cdot m$ vector. Assume $A$ is symmetric. Then $A$ is diagonalizable, i.e. there is an unitary matrix $P$ such that $P^{t} A P=\bar{\lambda}(A) I$, where $\bar{\lambda}(A)=\left(\lambda_{1}, \lambda_{2}, \cdots, \lambda_{n}\right)$ is the vector of eigenvalues of $A$, and we assume that $\lambda_{1} \geq \lambda_{2} \geq \cdots \lambda_{n}$.

An important relation between the effect of perturbing a matrix on its eigenvalues is the HoffmanWielandt inequality (see e.g. [Wi65]).

Theorem 6 (Hoffman-Weilandt Inequality) Let $A, B$ be matrices with $A-B$ symmetric. Then

$$
\|\lambda(A)-\lambda(B)\| \leq\|A-B\|
$$

Some $n \times n$ matrices we shall see later are:

- $H$ - the Sylvester matrix. Here $n=2^{k}$, and considering the indices as subsets of $[k], H_{i j}=(-1)^{i \cap j}$. For all $i \in[n],\left|\lambda_{i}(H)\right|=$ $\sqrt{n}$

- DFT - the Discrete Fourier Transform. Here $\omega$ is an $n$th primitive root of unity, and $D F T_{i j}=\omega^{i j}$. For all $i \in[n],\left|\lambda_{i}(D F T)\right|=$ $\sqrt{n}$

- $P P$ - the Projective Plane (see [Ha86]). Here $n=p^{2}+p+1$ for some prime $p$. PP is a Boolean matrix, with exactly $p+11$ 's in every row and column. For all $i \in[n]$, $\left|\lambda_{i}(P P)\right|=p \simeq n^{1 / 4}$

\section{$3 \quad$ Times-Space Tradeoffs}

In this section we show that there is no efficient analog to Theorem 1 of Baur and Strassen. In fact, the gap between $T S(f)$ and $T S(\Delta f)$ can be nearly linear - the worst possible. We exhinbit families of matrices $M$ for which $T S\left(l_{M}\right)$ is almost quadratic, while $T S\left(b_{M}\right)$ is nearly linear. The best gap we can achieve will depend on how large entries in $M$ are allowed to be (and hence on the size of the base field $F$ ), as well as how explicit the matrices are.

Lemma 2 Let $G(V, E)$ be any n-superconcentrator. Let $F$ be any field, and $H$ any finite subset of it. Let $w: E \rightarrow H$ be chosen uniformly at random. Then

1. The probability that all square minors in $M(G, w)$ are nonsingular is at least 1 $2 n 2^{2 n} /|H|$.

2. The probability that all square minors in $M(G, w)$ have at least half the maximum rank is at least $1-n^{3} /|H|$.

Proof: Let $V=I \cup R \cup O$ as before, the inpus, middle and output vertices of $G$, resp. Fix $s$ and an $s \times s$ minor $N$ of $M(G, w)$, say on rows $I^{\prime} \subseteq I$ and columns $O^{\prime} \subseteq O$. Since $G$ is a superconcentrator, there are $s$ vertex disjoint paths from $I^{\prime}$ to $O^{\prime}$. By renaming, we can assume wlog that $I^{\prime}$ (resp. $O^{\prime}$ ) are the first $s$ vertices of $I$ (resp. $O$ ), and moreover that the disjoint paths are formed by the edges $\left(I_{k}, R_{k}\right)$ and $\left(R_{k}, O_{k}\right)$ for all $1 \leq k \leq s$.

Fix the weights assigned by $w$ to all other edges in $E$ in an arbitrary way, and let $a_{k}$ (resp. $b_{k}$ ) be variables (indeterminates) whose value will be the weight assigned to $\left(I_{k}, R_{k}\right)\left(\operatorname{resp} .\left(R_{k}, O_{k}\right)\right)$. Call this set of $2 s$ variables $Z$. Thus our new probability space now assignes independent random weights from $H$ to $Z$.

Let $\hat{N}$ be our minor $N$ before assigning values to $Z$ so the entries $\hat{N}_{k, m}$ are polynomials over these variables. ¿From the definition of $M$ in subsection 2.5 the following should be clear.

Claim 1 - For every $1 \leq k \neq m \leq s, \hat{N}_{k, m}$ is a linear function. 
- For every $k, \hat{N}_{k, k}$ is a quadratic polynomial with exactly one monomial of degree 2 having a nonzero coefficient: the monomial $a_{k} b_{k}$.

Now we are ready to prove the two parts of the theorem, starting with part (1). Consider $\operatorname{det}(\hat{N})$, again as a polynomial in $F[Z]$. Clearly it is not identically zero, as the monomial $\prod_{k=1}^{s} a_{k} b_{k}$ has a nonzero coefficient, and is of degree $2 n$. By Theorem 5 we know that $\operatorname{Pr}[\operatorname{det}(N)=0]=$ $\operatorname{Pr}[\operatorname{det}(\hat{N}(w))=0] \leq 2 n /|H|$.

Since the bound above holds conditioned on every fixed choice of values $w$ assignes outside $Z$, it also holds over the whole probability space for this fixed minor $N$. Thus the final bound follows from the fact that there are $2^{2 n}$ minors.

Proving part (2) is slightly more complicated. Fix $N$, and values outside $Z$ again. We shall assign the values to $a_{k}, b_{k}$ for a single $k$ at a time, going from 1 to $s$. If $N_{k}$ denotes the $k \times k$ principal minor of $N$ (with $N_{0}=1$ ), we will prove:

Claim $2 \operatorname{Pr}\left[r k\left(N_{k}\right)=r k\left(N_{k-1}\right)\right] \leq 2 /|H|$, where the probability is taken for every fixed choice of $a_{1}, b_{1}, \cdots, a_{k-1}, b_{k-1}$, and a random choice of $a_{k}, b_{k}$.

Let us see why this implies part (2). Note that the probability that the event in the claim happens at least $s / 2$ times (which will cause $r k(N) \leq$ $s / 2)$ is at most $2^{s}(2 /|H|)^{s / 2}$. There are at most $n^{2 s}$ minors of size $s$, and it is easy to calculate the validity of the bound in (2) by summing this estimate over all $s \leq n$.

To prove the claim, assume $r k\left(N_{k-1}\right)=t$, and let $P$ be any $t \times t$ nonsingular minor of $N_{k-1}$ (taking $Q=N_{0}$ if $t=0$. Add to $P$ the (appropriate entries of) $k$ th row and column of $N$ (resp. $\hat{N}$ ), to create the matrix $Q$ (resp. matrix of variables $\hat{Q})$. It is clear that $\operatorname{Pr}\left[r k\left(N_{k}\right)=\right.$ $t]=\operatorname{Pr}[r k(Q)=t]=\operatorname{Pr}[\operatorname{det}(\hat{Q})=0]$. But observe that $\operatorname{det}(\hat{Q})$ is a quadratic polynomial in the two variables $a_{k}, b_{k}$. Moreover the only degree 2 monomial $\left(a_{k} b_{k}\right)$ has coefficient $\operatorname{det}(P)$ whcih is nonzero by the choice of $P$, so the claim follows again form Schwartz's lemma.

¿From Lemma 2, together with Theorem 2, Theorem 3, Lemma 1 and Proposition 1 we de- duce the main result of this section, namely a nearly linear gap between $T S(f)$ and $T S(\Delta f)$.

Theorem 7 For every $n$, if $|F|>n^{4}$, there is an $n \times n$ matrix $M$ with entries in $F$ satisfying:

- $L^{b}\left(b_{M}\right)=O\left(n(\log n)^{2}\right)$, and hence $T S\left(b_{M}\right)=$ $O\left(n(\log n)^{2}\right)$.

- $T S\left(l_{M}\right)=\Omega\left(n^{2}\right)$

The last theorem in this section achieves a similar gap can be obtained for small finite fields. We demonstarte it only for $F=G F(2)$.

Theorem 8 For every $n$, there is an $n \times n$ binary matrix $M^{\prime}$ so that the following bounds hold for computations both over $F=G F(2)$ and $F=$ $Q$.

- $L^{b}\left(b_{M^{\prime}}\right)=O\left(n(\log n)^{2}\right)$, and hence $T S\left(b_{M^{\prime}}\right)=$ $O\left(n(\log n)^{2}\right)$.

- $T S\left(l_{M^{\prime}}\right)=\Omega\left(n^{2} /(\log n)^{2}\right)$

Proof: For any $m$ set $t=4 \log m, n=m t$, and $G(V, E)$ a depth $2 m$-superconcentrator. By Theorem 2 there is an assignement $w: E \rightarrow$ $G F\left(2^{t}\right)$, such that for every $s \leq m$, every $s \times s$ minor of $M=M(G, w)$ has rank $\geq s / 2$ over the field $G F\left(2^{t}\right)$. Now define the $m \times n$ matrix $M^{\prime}$ over $G F(2)$, simply by expanding every entry in $M$ into its standard $t$-bit representation. The bounds in the theorem hold for this $M^{\prime}$.

To obtain the lower bound on $T S$ view the columns of $M^{\prime}$ as partitioned into $m$ blocks (each corresponding to an original column in $M$ ), of $t$ bits each. Now consider any $s \times(n-m / 2)$ minor of $M^{\prime}$. It must contain at least $m / 2$ complete blocks. Since the sum of any subset of these rows will yield $0^{t}$ in any complete block iff that sum yields 0 over $G F\left(2^{t}\right)$ in the corresponding column of $M$, we immediately see that the rank of this minor is at least $s / 2$ over $G F(2)$ (and thus also over $Q)$. Using the second part of Theorem 2, and $n=4 m \log m$ the lower bound on $T S\left(b_{M^{\prime}}\right)$ follow.

To obtain the upper bound on the formula size, consider now the columns of $M^{\prime}$ as partitioned to $t$ blocks (corresponding to each bit position in the $t$-bit representation of elements in 
$G F\left(2^{t}\right)$ ), of $m$ columns each. Call the $t$ matrices derived from this decomposition $M_{i}^{\prime}(1 \leq i \leq t)$. Let $w_{i}(e)$ be the $i$ th bit of the weight assigned by $w$ to the edge $e$ of $G$. It is clear that for every $i, M_{i}^{\prime}=M\left(G, w_{i}\right)$. But note that $L^{b}\left(b_{M^{\prime}}\right) \leq$ $\sum_{i=1}^{t} L^{b}\left(b_{M_{i}^{\prime}}\right) \leq t C(G)$. By taking $G$ from Theorem 3, and using $n=O(m \log m)$ again we get the upper bound.

Note: In describing the matrices $M(G, w)$ there were two nonconstructive parts: the superconcentrator $G$ (constructed probabilistically in Theorem 3) and $w$ that was chosen at random. As we have explicit superconcentrators that are not much larger (Theorem 4), it would be extremely interesting to find an explicit weight assignment $w$ to the edges of any depth 2 superconcentrator $G$, that will make all minors of $M(G, w)$ nonsingular. A way around this problem was suggested to us by Ben-Or: simply use new variables for the weights. This creates a polynomial of degree 4, but does establish the separation for explicit functions. Formally, combining this idea with Theorems 4, 8 gives:

Corollary 2 For every field there is a polynomial time algorithm that for every $n$ outputs a degree 4 polynomial $f_{n}$ on $n$ variables, satisfying $T S\left(f_{n}\right)=O\left(n^{1+o(1)}\right)$, and $T S\left(\Delta f_{n}\right)=\Omega\left(n^{2-o(1)}\right)$.

We conclude this section with a weak lower bound on time-space trade-offs. While weak, note that it implies that linear time algorithms require linear space.

Theorem 9 Let $M=M(G, w)$ be an $n \times n$ matrix with all minors nonsingular (over some field $F$ ). Assume $A$ is a bilinear straight line program (over the same field $F$ ) computing $b_{M}$, with $T(A)=T$ and $S(A)=S$. Then $2^{T / n} T S=$ $\Omega\left(n^{2}\right)$.

Proof: Let $T=n k / 4$. The straight line program $A$ defines a sequence $\sigma$ of variables from $\left\{x_{1}, \cdots, x_{n}, y_{1}, \cdots, y_{n}\right\}$ of length at most $n k / 4$, in the order they appear in $A$. By the main lemma in [AM88], there are subsets $X_{0}, Y_{0}$ of the $x$ and $y$ variables, respectively, such that $\left|X_{0}\right|=\left|Y_{0}\right|=n /\left(2^{k}\right)$ satisfying the following property: if we remove all other variables from $\sigma$, the resulting subsequence has at most $k$ alternations between $x$ and $y$ variables.

Setting the remaining variables to 0 , we get a reduced program $A_{0}$ computing the bilinear form on the variables $X_{0}, Y_{0}$, defined by the submatrix $M_{0}$ of $M$ on rows $X_{0}$ and columns $Y_{0}$. Note that $r k\left(M_{0}\right)=n /\left(2^{k}\right)$. Assume wlog that $A$ uses a different set of registers for linear forms of the $x$ 's (say $R_{X}$ ) and of the $y$ 's (say $R_{Y}$ ). Then $A_{0}$, by the property above, can be partitioned into $k$ "stages" such that in any $x$-stage multiplications can involve only registers from $R_{Y}$ (but not $y$ variables), and conversely for $y$-stages.

The lower bound now follows from the following easy facts.

1. Every multiplication gate creates one bilinear form of rank 1.

2. Since $\left|R_{Y}\right| \leq S$, the dimension of the span of rank 1 bilinear forms generated in one $x$-stage is at most $S$ (and analogously for $y$-stages).

3. The rank of the output of $A_{0}$ is at most the dimension of the span of all rank 1 bilinear forms it computes.

4. Rank is subadditive, so the total dimension is at most $S k$, yielding $S k \geq n /\left(2^{k}\right)$.

\section{Circuits and formulae with small constants}

For this section we restrict attention to the real and complex number fields. We impose the following restriction on our circuit: any plus gate computes a linear combination of its inputs, with coefficients of absolute value at most 1 . For any polynomial $f$, (and similarly for a set of polynomials) denote by $C_{1}(f)$ and $L_{1}(f)$ the size of the smallest circuit and formula computing $f$. We note that the analogs of Propositions 2, 3 hold for $C_{1}$ as well.

As we look at asymptotic complexity, the choice of bound 1 is clearly arbitrary, and can be replaced by any other constant. Also, it is clearly 
interesting to study $C_{1}$ and $L_{1}$ only for polynomials with small coefficients. There are no examples of such polynomials for which the restricted model is significantly weaker than the general one. This question becomes especially interesting, since nontrivial lower bounds can be proved in the restricted model.

The first to prove such lower bounds was Morgenstern [Mo73]. He observed that the standard FFT algorithm for the Discrete Fourier Transform works in the restricted model. If $D F T$ denotes the $n \times n$ DFT matrix, then $C_{1}\left(l_{D F T}\right) \leq$ $\frac{1}{2} n \log n$. Morgenstern showed that this is best possible.

Theorem 10 [Mo73] $C_{1}\left(l_{D F T}\right) \geq \frac{1}{2} n \log n$

The proof uses a very elegant volume argument. In fact, his proof yield, for any matrix $M$, the lower bound

Theorem $11 C_{1}\left(l_{M}\right) \geq \log |\operatorname{det}(M)|$

Note that $\operatorname{det}(M)=\Pi_{i} \lambda_{i}$, where the $\lambda_{i}$ 's are the eigenvalues of $M$. For the DFT and Hadamard mtrices all eigenvalues are $\sqrt{n}$ in absolute value.

Very recently Chazelle [Ch94] used more refined entropy argumnets to derive similar bounds in a slightly stronger model, for problems arising from 2-dimentional range-query computations.

An immediate question, is whether such lower bounds can be proved for one function (rather then a set of functions). The immediate answer follows from observing that the construction of Baur and Strassen (Theorem 1) does not introduce any new constants (except 1). This implies the following circuit lower bounds on explicit bilinear functions. Corollary 3 For every $M C_{1}\left(b_{M}\right)=\Omega(\log |\operatorname{det}(M)|) \cdot$ lemma. In particular, $C_{1}\left(b_{D F T}\right), C_{1}\left(b_{H}\right), C_{1}\left(b_{P P}\right)=\Omega(n \log n)$.

Next we turn attention to formula size of bilinear functions. We again stress that in the unrestricted model, the only nontrivial lower bound on explicit bilinear forms is the the $\Omega(n \log n)$ bound in the end of the previous section. For the restricted model, such a lower bound trivially follows the circuit lower bound above. However, we can do much better, in the main theorem of this section.

Theorem $12 L_{1}^{b}\left(b_{H}\right)=\Omega\left(n^{\frac{5}{4}}\right), L_{1}^{b}\left(b_{P P}\right)=\Omega\left(n^{\frac{9}{8}}\right)$

Comments While we cannot obtain a general clean expression for the formula lower bound in the above Theorem 12, the proof will reveal that the clean form for the examples we have is

$$
L_{1}^{b}\left(b_{A}\right)=\Omega\left(\sum_{i} \sqrt{\left|\lambda_{i}(A)\right|}\right)
$$

There are a few things to note with respect to this form.

- It is very interesting to compare it to the circuit size lower bounds in Theorem 11 and Corollary 3 , in their dependence on the spectrum of the matrix, namely

$$
C_{1}\left(b_{A}\right)=\Omega\left(\sum_{i} \log \left|\lambda_{i}(A)\right|\right)
$$

- It will be clear from the proof below that a similar formula lower bound can be proved for other "regular" matrices $A$. These include, for example, all incidence matrices of points vs. subspaces in projective geometries, and more generally block designs [Ha86].

- This form of a formula lower bound is tight in general. For any diagonal matrix $A$ it can be easily seen that $L_{1}^{b}\left(b_{A}\right)=O\left(\sum_{i} \sqrt{\left|\lambda_{i}(A)\right|}\right)$.

- Recently [Lo95] generalized our proof and was also able to obtain size-depth tradeoffs for the the liner function $l_{A}$.

Now we return to the proof of Theorem 12 . Before proving it, we need the following simple

Lemma 3 For every $U, V$ matrices such that $U V$ diagonalizable,

$$
\|U\|^{2}+\|V\|^{2} \geq 2|\lambda(U V)|
$$

Proof: First we observe that without loss of generality, $U V=\lambda(U V) I$. (Otherwise, let $P$ be the unitary matrix for which $P U V P=\lambda(U V) I$, 
and set $U^{\prime} \leftarrow P U, V^{\prime} \leftarrow V P$ and note that $\left\|U^{\prime}\right\|=\|U\|$ and $\left.\left\|V^{\prime}\right\|=\|V\|.\right)$ The rest follows from the basic fact that $a^{2}+b^{2} \geq 2 a b$ for any two reals $a, b$.

Proof: (of Theorem 12) Assume that $L_{1}^{b}\left(b_{A}\right) \leq$ $\frac{1}{10} n d$, for some $n \times n$ diagonalizable matrix $A$ and some parameter $d$. Then by the decomposition of Equation 2, and the fact that the formula uses only bounded constants, $\exists U, V$ s.t. $U V=A$ and $|U|+|V| \leq \frac{1}{10} n d$. Let $S_{U}$ (resp. $\left.S_{V}\right)$ be the set of rows of $U$ (resp. columns of $V$ ) having entries larger than $d$ in absolute value. Clearly, $\left|S_{U}\right|,\left|S_{V}\right| \leq \frac{n}{10}$, so $S=S_{U} \cup S_{V}$ satisfies $|S| \leq \frac{n}{5}$.

Let $U^{\prime}$ (resp. $V^{\prime}$ ) be the matrix $U$ (resp. $V$ ) in which we replace the rows (resp. columns) of $S$ by zeros. Thus $\left|U^{\prime}\right|_{\infty},\left|V^{\prime}\right|_{\infty} \leq d$ and with the bound above we have $\left\|U^{\prime}\right\|^{2}+\left\|V^{\prime}\right\|^{2} \leq n d^{2}$.

Let $A^{\prime}=U^{\prime} V^{\prime}$. Note that $A$ and $A^{\prime}$ differ only in the rows and columns indexed by $S$. Thus we have:

- For $A=H,\left\|A-A^{\prime}\right\|^{2} \leq \frac{2}{5} n^{2}$

- For $A=P P,\left\|A-A^{\prime}\right\|^{2} \leq \frac{2}{5} n^{3 / 2}$

By the Hoffman-Wielandt inequality, and the properties of these matrices listed at the end of section 2.7, at most $\frac{4}{5}$ of the $\lambda_{i} \mathrm{~s}$ in $H^{\prime}$ (resp. $\left.P P^{\prime}\right)$ differ from the corresponding ones in $H$ (resp. $P P$ ) by more than $\sqrt{n / 2}$ (resp. $(n / 2)^{\frac{1}{4}}$ ; note that this bound uses the fact that every row and column in $P P$ has only $O(\sqrt{n}) 1$ 's $)$. Thus $\left|\lambda\left(H^{\prime}\right)\right|=\Omega\left(n^{3 / 2}\right)$, and $\left|\lambda\left(P P^{\prime}\right)\right| \Omega\left(n^{5 / 4}\right)$. By Lemma 3, we are done.

\section{Acknowledgements}

We are greatful to Allan Borodin for motivating us to look at time-space trade-off question, as well as for many illuminating converstions. We also thank Michael Ben-Or for helpful comments on an earlier version of this paper.

\section{References}

[AM88] N. Alon and W. Maass, "Meanders and their applications in lower bounds arguments", JCSS, 37, pp. 118-129, 1988.
[Bo94] A. Borodin, Private communication, 1994.

[BS82] W. Baur, V. Strassen, "The complexity of partial derivatives", Theoretical Computer science, 22, pp. 317-330, 1982.

[Ch94] B. Chazelle, "A Spectral Approach to Lower Bounds", Proc. of the 35th FOCS, 1994, to appear.

[DDPW] D. Dolev, C. Dwork, N. Pippenger, and A. Wigderson, "Superconcentrators, Generalizers, and Generalized Connectors with Limited Depth," Proc. of the 15th STOC, pp. 42-51, 1983.

[Ha86] M. Hall, Combinatorial Theory, John Wiley, 1986.

[Lo95] S. V. Lokam, "Size-depth tradeoffs for linear transformations", manuscript, 1995.

[Mo73] J. Morgenstern, "Note on a lower bound of the linear complexity of the fast Fourier transform", JACM 20 (2), pp. 305-306, 1973.

[Mo85] J. Morgenstern, "How To Compute Fast A Function And All Its Derivatives", SIGACT News, 16 (4), pp. 60-62, Spring 1985.

[Pi78] N. Pippenger, "Superconcentrators", SIAM J. Comput. 6, pp. 298-304, 1978.

[Pi82] N. Pippenger, "Superconcentrators of Depth 2," J. Comp. and Sys. Sci. 24, pp. 82-90, 1982.

[Sc80] J. T. Schwartz, "Fast Probabilistic Algorithms for Verification of Polynomial Identities", JACM 27 (4), pp. 701-717, 1980 .

[St73] V. Strassen, "Die Berechnungskomplexitat vo elementarsymmetrischen Funktionen und von Interpolationskoefizienten", Numer. Math. 20, pp. 238-251, 1973. 
[SZ94] A. Srinivasan and D. Zuckerman, "Computing with Very Weak Random Sources," Proc. of the 35th FOCS, 1994, to appear.

[To80] M. Tompa, "Time Space Tradeoffs for Computing Functions, Using Connectivity Properties of Their Circuits," $J$. Comp. and Sys. Sci, 20, pp. 118-132, 1980.

[Va76] L.G. Valiant, "Graph Theoretic Properties in Computational Complexity," $J$. Comp. and Sys. Sci. 13, pp. 278-285, 1976.

[Wi65] J. H. Wilkinson, The Algebraic Eigenvalue Problem, Oxford University Press, pp. 104-109, 1965.

[WZ93] A. Wigderson, D. Zuckerman, "Expanders that Beat the Eigenvalue Bound, Explicit Construction and Applications", Proc. of the 25th STOC, pp. 245-251, 1993. 